\title{
The Medical Home Concept and Congenital Adrenal Hyperplasia: A Comfortable Habitat!
}

\author{
Selma Feldman Witchel \\ Division of Pediatric Endocrinology, Children's Hospital of Pittsburgh of UPMC, University of Pittsburgh, Pittsburgh, PA 15224, USA
}

Correspondence should be addressed to Selma Feldman Witchel, selma.witchel@chp.edu

Received 17 March 2010; Accepted 29 March 2010

Academic Editor: Peter Allen Lee

Copyright (๑) 2010 Selma Feldman Witchel. This is an open access article distributed under the Creative Commons Attribution License, which permits unrestricted use, distribution, and reproduction in any medium, provided the original work is properly cited.

\begin{abstract}
Patient-centered interdisciplinary health care for children with chronic medical disorders represents an evolution from the traditional "stop and go" treatment for acute illnesses. This model for health care delivery has been called the "medical home," a concept that was originally developed in pediatrics for the care of children with special needs. Patient and family-centered, comprehensive, interdisciplinary, culturally effective, and readily accessible health care delivery is desirable for the care of children with congenital adrenal hyperplasia. As children with congenital adrenal hyperplasia (CAH) become adolescents and young adults, transfer of this health care delivery model to adult endocrinologists is appropriate.
\end{abstract}

\section{Introduction}

Historically, medical care systems were developed and organized to diagnose and treat acute illnesses or injuries. Health care professionals, patients, and families focused on rapid identification and resolution of the acute medical problem with infectious diseases being the prototype for this type of patient-physician interaction. Thus, mechanisms to promote and educate patient and family regarding self-management skills were not considered to be essential.

With improved treatments, children with chronic illnesses now have increased survival rates. Challenges for children with chronic illnesses and their parents include dealing with symptoms, psychosocial and emotional impact, daily medications and treatments, lifestyle changes, and regular school absences for physician visits and acute medical issues [1]. The traditional "stop and go" approach to medical care encourages fragmented health care which is inefficient, expensive, and suboptimal for the care of children with chronic medical conditions such as congenital adrenal hyperplasia.

\section{The Medical Home}

The term "medical home" first appeared in a book written by the American Academy of Pediatric Council on Pediatric Practice (COPP) which emphasized the importance of a single repository for a child's medical records [2]. In 1977, the American Academy of Pediatrics issued a statement that quality medical care is best provided when all of the child's medical data are together in one place, (a medical home) and these data are readily accessible to the responsible physician or physicians [3]. Over time, this concept has evolved from merely centralized medical records to a system involving comprehensive, compassionate, well-coordinated, culturally effective, holistic, and readily accessible patient and family-centered health care in partnership with well-trained physicians and ancillary health care personnel who provide continuity of care [4].

One goal of the Healthy People 2010 for Children with Special Health Care Needs is regular ongoing comprehensive care in a medical home [5]. The Healthy People 2010 initiative is sponsored by the federal government and coordinated by The Office of Disease Prevention and Health Promotion, U.S. Department of Health and Human Services to promote 
national health and disease prevention. The first goal of this program is to increase life expectancy and improve quality of life for all (http://www.health.gov/healthypeople/). Data collected through the 2001 National Survey of Children with Special Health Care Needs revealed that children lacking a medical home were more likely to delay, forgo, or completely lack needed health care [6]. Additional analyses of data collected through this survey concluded that a sense of partnership was associated with improved outcomes across a number of important health care measures such as missed school days, access to specialty care, and satisfaction with care [7]. For adults with chronic illnesses such as type 2 diabetes mellitus, evidence-based data developed with support from the Robert Wood Johnson Foundation has demonstrated improved medical management in the primary care setting in the context of a medical home [8].

Despite current discussions regarding the optimal care delivery systems, relationships among health care providers, and the economics of health care delivery, the foundation of the medical home is the individualized care plan which is tailored to the specific needs of each patient and evolves as the patient's needs change. Implementation involves an interdisciplinary team approach. Care is coordinated to encompass planned visits, monitoring of disease markers, delivery of preventive care, provision of anticipatory guidance, and encouragement of self-management. Within the framework of the medical home, the focus of care shifts from acute illness management to an emphasis on developmental perspectives and anticipatory guidance targeted for the needs of each individual. Thus, a medical home provides proactive rather than reactive health care [9].

The American Academy of Pediatrics and the American Academy of Family Physicians endorse the medical home concept. Specific elements of the medical home include the first-responder, continuity, comprehensively coordinated care, communication, thorough medical records, and patient advocacy. Although originally described as important for primary care pediatrics, these elements are relevant for the care of children with congenital adrenal hyperplasia $(\mathrm{CAH})$ and other chronic illnesses or other life-long conditions that require long-term multidisciplinary care. Implementation of these elements encourages family-centeredness and cultural sensitivity.

For children with $\mathrm{CAH}$, the primary care physician (PCP), the pediatric endocrinologist, pediatric urologist/surgeon, and other health care providers can and should collaborate to develop partnerships with the child and the family. The PCP retains responsibility for monitoring general health and well-being, provision of immunizations, and care for acute illnesses. The subspecialists and their ancillary staffs can monitor and coordinate needs for the chronic medical condition. Children and families can only benefit when necessary services are integrated and coordinated. Specific characteristics and responsibilities of these partnerships evolve as the children grow and mature.

What characteristics contribute to effective management of chronic disorders or other life-long conditions in children? Successful models for care of patients with chronic medical disorders include several features. Features of these systems include explicit protocols, time management to accommodate more complex patients, attention to patient information needs and behavioral changes, access to requisite expertise, information technology, and planned transitions from pediatric to adult health care providers [10]. Continuity of care, individualized recommendations, evidence-based treatments, collaboration among health care professionals, patient/family education, patient empowerment, advocacy, and quality assurance are also essential elements. Electronic medical records with the capability to provide written instructions, reminders for upcoming office visits, electronic exchange of information among health care providers, and a "tickler file" to detect overdue services enhance communication.

The medical home model encourages identification of the individual's strengths, his/her educational needs regarding the pathophysiology of $\mathrm{CAH}$, and his/her understanding of future health care needs. Other factors considered in this model include the complexity of the medical illness, cognitive abilities, mental health status, family support, and need for surgery. The report of the Congenital Adrenal Hyperplasia Research Education \& Support (CARES) Foundation meeting on Comprehensive Care for Children with $\mathrm{CAH}$ provides the initial roadmap for interdisciplinary care or "medical homes" for children with CAH. The CARES Foundation report represents the work product of parents and physicians to design the ideal comprehensive clinical setting for children with CAH. The guidelines for the patients with CAH developed by The Endocrine Society Taskforce provide more specific medical management recommendations [11]. Thus, the concept of a medical home in the context of a multidisciplinary patient-centered health care facility is hopefully on the road to implementation.

As pediatric healthcare providers and parents, our vision is that our patients with $\mathrm{CAH}$ will become productive responsible citizens in our society [12]. The pediatric medical home provides a caring environment that can equip our patients with the knowledge and skills necessary to achieve this goal. One accomplishment along the pathway to fulfill this objective is the "transition" to adult care providers [1315]. Although analogous to "graduation", transition should be considered as a purposeful, planned transfer of care from pediatric healthcare providers to adult care providers. This process should consider medical, psychosocial, educational, cognitive, emotional, and vocational needs. For adolescents undergoing transition to adult care providers, pretransition planning including an assessment of readiness is crucial. Expectations and differences in health care delivery systems between pediatric and adult care systems should be discussed. Following transition to adult care providers, the concept of the medical home can continue. Even though medical goals may shift from the focus on linear growth and "on-time" puberty to concerns related to fertility, sexuality, bone health, and cardiovascular disease risks, the paradigm of comprehensive, multidisciplinary, compassionate, well coordinated, culturally effective, accessible patient and family-centered health care persists [16].

Despite the exhilaration of this major life event, numerous barriers can confound a smooth transition. Pediatricians 

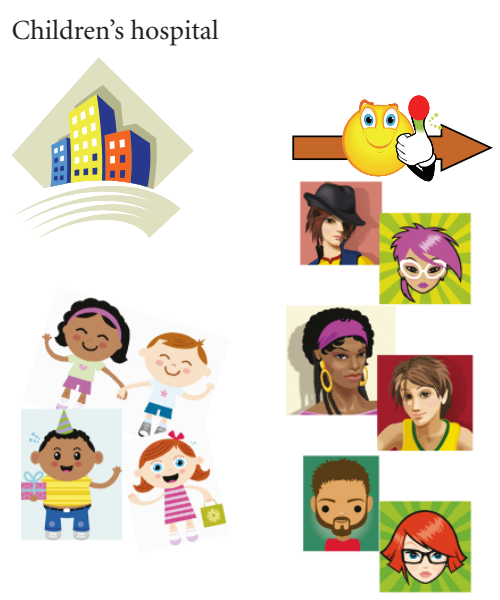

Adult medical office
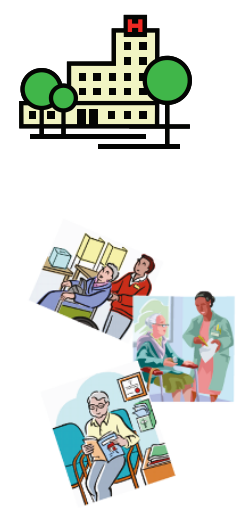

Figure 1: An adolescent's perspective on pediatric and adult medical offices. Adolescents often feel out of place in waiting rooms with babies and young children. Prior to transfer to adult care providers, their impression of waiting rooms for adult endocrinology practices may be distorted.

and staff may have difficulty "letting go" and transferring this long-term relationship to adult healthcare providers. Adolescents may feel that they stick out like "sore thumbs" in both pediatric and adult waiting rooms (Figure 1). Overinvolvement of parents can lead to adolescents feeling excluded and thwarted from participating in their own health care. This can undermine the adolescent's emerging autonomy and self-responsibility [17]. Parents need to make the transition from being the "CEO" of their child's health care to becoming the consultant, and eventually, to a bystander while the child ascends from the consumer to the "CEO" [18]. This transfer of health care power and control is often difficult for the parents as their role and influence decrease.

Normal adolescent development with tendencies to question or reject advice and the tendency to not fully comprehend the consequences of his/her actions also contributes to these barriers [19]. Patient behaviors such as texting and playing games on cell phones, increased risktaking behavior, poor adherence to recommendations, and disinterest in participating in discussions frustrate health care providers and parents. Another biologic influence is that structural and functional brain imaging demonstrates that maturational changes of the areas involved in executive functioning may continue into early adulthood [20]. The well-established relationships between the health care team and the adolescent patient in the context of the medical home can provide a safety net for the patient. In this setting, the adolescent may feel comfortable sharing private concerns, worries, scary thoughts, and other anxieties with the supportive, caring, and trustworthy health care personnel that he or she has known for many years.

System issues relevant to the transition process include patchy health care insurance because the young adult may no longer be eligible for coverage by parents' insurance plan(s) and/or they may be denied coverage to due to preexisting conditions. At times, medical services seem to be fragmented, require multiple different forms and insurance information, and communication between the various health care providers is incomplete [21]. The medical home can facilitate completion of various forms and provide better communication among health care providers.

Adult health care providers assume that their adolescent and young adult patients are active partners who are knowledgeable about their disorder, are autonomous, and are capable of negotiating the health care system. These expectations differ from the situation with pediatric health care providers where parents assume a greater role in management of chronic health issues. Physician and patient make management decisions together with expectation that the patient will adhere to the treatment plan. Adolescents especially those with chronic disorders may be unprepared and unready to participate as an active partner in their own health care. Thus, there is reconfiguration of doctorparent-patient relationships to doctor-patient relationship. Suboptimal transitions can lead to mediocre connections with adult health care providers which may ultimately culminate in "drop-out" from health care.

Knowledge about self-management does not always predict good adherence. For some adolescents and young adults, nonadherence to recommendations may represent a conscious decision to minimize the intrusiveness of the disease. Problem solving or brainstorming discussions to find creative solutions regarding adherence to the recommended management regimens and other aspects of their lives may be invaluable. Thus, continuity of the adult care provider may encourage such discussions because the adolescent may feel more comfortable to share decision-making process in this situation.

The literature regarding transition for children with diabetes mellitus, cystic fibrosis, rheumatologic disorders, sickle cell anemia, and solid organ transplants is expanding [2225]. What can we learn from their experiences? Available data and feedback suggest that transition is a planned process. Themes that emerged from one small study of children who had undergone heart transplant were apathy among the children, anxiety among the parents, and need for collaboration and communication between pediatric and adult centers [26]. The report of the 2008 consensus conference regarding transition of adolescents with solid organ transplant offers many suggestions which are applicable to adolescents with endocrine disorders (Table 1). One suggestion is a transition clinic where both adult and pediatric health care providers are available [27].

Traditionally, curricula for teaching how to coordinate chronic care among health care providers are limited [28, 29]. Most care coordination is learned and developed on an ad hoc basis. Development and functioning as an effective high-performance team will require education of health care providers [30]. Provision of training for health care providers and ancillary support staff can only enhance the effectiveness of coordinated care within a medical home. Most adult medicine training programs, residency or adult endocrinology, lack specific instruction regarding conditions with pediatric onset. In the absence of appropriate primary 
TABLE 1: Milestones for transfer to adult care.

(1) Understanding etiology, basic genetics, pathophysiology of congenital adrenal hyperplasia

(2) Understanding hormone replacement therapies and knowledge of sick day/stress dose management

(3) Understanding impact of CAH on sexuality and reproductive health

(4) Demonstrating responsibility for own healthcare

(a) Knowledge of medications

(b) Calling for own prescription refills and scheduling office visits

(c) Independently communicating with health care providers

(d) Knowledge regarding when to seek emergency medical attention

(e) Comprehension of health insurance coverage and requirement

(5) Readiness to transition to adulthood by attending college or entering the workforce

(6) Ownership of his/her medical history in a concise format

(7) Preparation of parents for their new role as spectators/advisors

care and specialized adult services, adolescents with chronic medical conditions risk dropping out of health care altogether, with the potential for dire health consequences. This shortage of adult expertise to care for young adults with childhood conditions is now being recognized and hopefully will be rectified [31].

The expansion and integration of transition strategies into the fabric of patient care offer the opportunity to assess outcome measures and identify quality benchmarks. Welldesigned comprehensive multicenter registries can collect the data to provide evidence-based outcome information. Two major types of data can be obtained. One category involves the structure and functioning of the medical home for the patients with CAH. The other category could include longitudinal outcome data collected through patient registries. Ideally, registries for CAH can be coordinated within the framework created by the Long-term Follow-up Committee of the Advisory Committee on Heritable Disorders in Newborns and Children (ACHDNC) [32]. Analyses of longitudinal data collected through a registry could provide information on relevant issues such as reproductive health, sexuality, incidence of testicular adrenal rest tumors, incidence of adrenal incidentalomas and adrenal myelolipomas, risk of acute adrenal insufficiency among individuals with $\mathrm{NCAH}$, bone health, metabolic consequences, and quality of life.

\section{Conclusion}

The medical home can provide comprehensive, holistic, interdisciplinary, patient and family-centered care throughout the lifecycle. The major goals of a medical home are to provide quality healthcare and meet the needs of children with $\mathrm{CAH}$ and their families such that the children with $\mathrm{CAH}$ experience good quality of life and develop into productive members of our society.

\section{References}

[1] E. H. Wagner, B. T. Austin, C. Davis, M. Hindmarsh, J. Schaefer, and A. Bonomi, "Improving chronic illness care: translating evidence into action," Health Affairs, vol. 20, no. 6, pp. 64-78, 2001.

[2] American Academy of Pediatrics, Council on Pediatric Practice, "Pediatric records and a "medical home"”, in Standards of Child Care, pp. 77-79, American Academy of Pediatrics, Evanston, Ill, USA, 1967.

[3] American Academy of Pediatric, Committee on Pediatric Emergency Medicine, "Advocating for ems-c on a broader scale," in Emergency Medical Services for Children: The Role of the Primary Care Provider, pp. 117-123, American Academy of Pediatrics, Elk Grove Village, Ill, USA, 1992.

[4] C. Sia, T. F. Tonniges, E. Osterhus, and S. Taba, "History of the medical home concept," Pediatrics, vol. 113, no. 5, pp. 14731478, 2004.

[5] US Department of Health and Human Services, Healthy People 2010: Understanding and Improving Health, Washington, DC, USA, Government Printing Office, 2nd edition, 2000.

[6] B. Strickland, M. McPherson, G. Weissman, P. van Dyck, Z. J. Huang, and P. Newacheck, "Access to the medical home: results of the national survey of children with special health care needs," Pediatrics, vol. 113, no. 5, supplement, pp. 14851492, 2004.

[7] D. Denboba, M. G. McPherson, M. K. Kenney, B. Strickland, and P. W. Newacheck, "Achieving family and provider partnerships for children with special health care needs," Pediatrics, vol. 118, no. 4, pp. 1607-1615, 2006.

[8] E. H. Wagner, L. C. Grothaus, N. Sandhu, et al., "Chronic care clinics for diabetes in primary care: a system-wide randomized trial," Diabetes Care, vol. 24, no. 4, pp. 695-700, 2001.

[9] W. C. Cooley, "Redefining primary pediatric care for children with special health care needs: the primary care medical home," Current Opinion in Pediatrics, vol. 16, no. 6, pp. 689692, 2004.

[10] E. H. Wagner, C. Davis, J. Schaefer, M. von Korff, and B. Austin, "A survey of leading chronic disease management programs: are they consistent with the literature?" Managed Care Quarterly, vol. 7, no. 3, pp. 56-66, 1999.

[11] P. W. Speiser, et al., "Congenital adrenal hyperplasia due to steroid 21-hydroxylase deficiency: an Endocrine Society Clinical Practice Guideline," Journal of Clinical Endocrinology and Metabolism. In press.

[12] I. A. Hughes, "Congenital adrenal hyperplasia: transitional care," Growth Hormone and IGF Research, vol. 14, supplement A, pp. S60-S66, 2004. 
[13] B. Kruse, F. G. Riepe, N. Krone, et al., "Congenital adrenal hyperplasia-how to improve the transition from adolescence to adult life," Experimental and Clinical Endocrinology and Diabetes, vol. 112, no. 7, pp. 343-355, 2004.

[14] G. S. Conway, "Congenital adrenal hyperplasia: adolescence and transition," Hormone Research, vol. 68, supplement 5, pp. 155-157, 2007.

[15] S. D. Grosse, M. S. Schechter, R. Kulkarni, M. A. LloydPuryear, B. Strickland, and E. Trevathan, "Models of comprehensive multidisciplinary care for individuals in the United States with genetic disorders," Pediatrics, vol. 123, no. 1, pp. 407-412, 2009.

[16] C. M. Ogilvie, N. S. Crouch, G. Rumsby, S. M. Creighton, L. M. Liao, and G. S. Conway, "Congenital adrenal hyperplasia in adults: a review of medical, surgical and psychological issues," Clinical Endocrinology, vol. 64, no. 1, pp. 2-11, 2006.

[17] D. Rosen, "Between two worlds: bridging the cultures of child health and adult medicine," Journal of Adolescent Health, vol. 17, no. 1, pp. 10-16, 1995.

[18] G. M. Kieckhefer and C. M. Trahms, "Supporting development of children with chronic conditions: from compliance toward shared management," Pediatric Nursing, vol. 26, no. 4, pp. 354-363, 2000.

[19] J. E. McDonagh and M. Kaufman, "The challenging adolescent," Rheumatology, vol. 48, no. 8, pp. 872-875, 2009.

[20] J. N. Giedd, "The teen brain: insights from neuroimaging," Journal of Adolescent Health, vol. 42, no. 4, pp. 335-343, 2008.

[21] A. M. Kelly, B. Kratz, M. Bielski, and P. M. Rinehart, "Implementing transitions for youth with complex chronic conditions using the medical home model," Pediatrics, vol. 110, no. 6, part 2, pp. 1322-1327, 2002.

[22] D. Allen and J. Gregory, "The transition from children's to adult diabetes services: understanding the "problem"', Diabetic Medicine, vol. 26, no. 2, pp. 162-166, 2009.

[23] M. S. Schechter and P. Margolis, "Improving subspecialty healthcare: lessons from cystic fibrosis," Journal of Pediatrics, vol. 147, no. 3, pp. 295-301, 2005.

[24] M. McPherson, L. Thaniel, and C. P. Minniti, "Transition of patients with sickle cell disease from pediatric to adult care: assessing patient readiness," Pediatric Blood and Cancer, vol. 52, no. 7, pp. 838-841, 2009.

[25] L. K. Tuchman, G. B. Slap, and M. T. Britto, "Transition to adult care: experiences and expectations of adolescents with a chronic illness," Child: Care, Health and Development, vol. 34, no. 5, pp. 557-563, 2008.

[26] S. J. Anthony, M. Kaufman, A. Drabble, M. Seifert-Hansen, A. I. Dipchand, and K. Martin, "Perceptions of transitional care needs and experiences in pediatric heart transplant recipients," American Journal of Transplantation, vol. 9, no. 3, pp. 614-619, 2009.

[27] L. E. Bell, S. M. Bartosh, C. L. Davis, et al., "Adolescent transition to adult care in solid organ transplantation: a consensus conference report," American Journal of Transplantation, vol. 8, no. 11, pp. 2230-2242, 2008.

[28] H. Holman, "Chronic disease-the need for a new clinical education," Journal of the American Medical Association, vol. 292, no. 9, pp. 1057-1059, 2004.

[29] H. R. Holman, "The inadequacy of medical education," Chronic lllness, vol. 5, no. 1, pp. 18-20, 2009.

[30] A. K. Jain, J. M. Thompson, J. Chaudry, S. McKenzie, and R. W. Schwartz, "High-performance teams for current and future physician leaders: an introduction," Journal of Surgical Education, vol. 65, no. 2, pp. 145-150, 2008.
[31] S. M. Sawyer, S. Drew, M. S. Yeo, and M. T. Britto, "Adolescents with a chronic condition: challenges living, challenges treating," The Lancet, vol. 369, no. 9571, pp. 1481-1489, 2007.

[32] A. R. Kemper, T. L. Trotter, M. A. Lloyd-Puryear, P. Kyler, W. G. Feero, and R. R. Howell, "A blueprint for maternal and child health primary care physician education in medical genetics and genomic medicine: recommendations of the United States secretary for health and human services advisory committee on heritable disorders in newborns and children," Genetics in Medicine, vol. 12, pp. 77-80, 2010. 